OPEN ACCESS

Edited by:

Akio Adachi,

Kansai Medical University, Japan

Reviewed by:

Amy Sims,

Pacific Northwest National Laboratory (DOE), United States

Yongwen Chen,

Third Military Medical University,

China

*Correspondence:

Enrique Santamaría

esantamma@navarra.es

Specialty section: This article was submitted to Virology,

a section of the journal

Frontiers in Microbiology

Received: 02 June 2020

Accepted: 10 August 2020

Published: 22 September 2020

Citation:

Lachén-Montes M, Corrales FJ,

Fernández-Irigoyen J and

Santamaría E (2020) Proteomics Insights Into the Molecular Basis of

SARS-CoV-2 Infection: What We Can

Learn From the Human

Olfactory Axis.

Front. Microbiol. 11:2101.

doi: 10.3389/fmicb.2020.02101

\section{Proteomics Insights Into the Molecular Basis of SARS-CoV-2 Infection: What We Can Learn From the Human Olfactory Axis}

\author{
Mercedes Lachén-Montes ${ }^{1,2,3}$, Fernando J. Corrales ${ }^{3,4}$, Joaquín Fernández-Irigoyen ${ }^{1,2,3}$ and \\ Enrique Santamaría, ${ }^{1,23 *}$
}

${ }^{1}$ Clinical Neuroproteomics Unit, Proteomics Platform, Navarrabiomed, Complejo Hospitalario de Navarra (CHN), Universidad Pública de Navarra (UPNA), Pamplona, Spain, ${ }^{2} / d i S N A$, Navarra Institute for Health Research, Pamplona, Spain, ${ }^{3}$ ProteoredInstituto de Salud Carlos III (ISCIII), Madrid, Spain, ${ }^{4}$ Proteomics Unit, National Centre for Biotechnology, Madrid, Spain

Like other RNA viruses, severe acute respiratory syndrome coronavirus 2 (SARS-CoV-2) replicates in host cells, continuously modulating the molecular environment. It encodes 28 multifunctional proteins that induce an imbalance in the metabolic and proteostatic homeostasis in infected cells. Recently, proteomic approaches have allowed the evaluation of the impact of SARS-CoV-2 infection in human cells. Here, we discuss the current use of proteomics in three major application areas: (i) virus-protein interactomics, (ii) differential proteotyping to map the virus-induced changes in different cell types, and (iii) diagnostic methods for coronavirus infectious disease 2019 (COVID-19). Since the nasal cavity is one of the entry sites for SARS-CoV-2, we will also discuss the potential application of olfactory proteomics to provide novel insights into the olfactory dysfunction triggered by SARS-CoV-2 in patients with COVID-19.

Keywords: proteomics, coronavirus infectious disease 2019, severe acute respiratory syndrome coronavirus-2, smell, mass-spectrometry

\section{INTRODUCTION}

Since the outbreak of the pandemic in Wuhan (China) in December 2019, coronavirus infectious disease 2019 (COVID-19) has spread globally, causing a crisis not only in our healthcare systems but also in the world economy (Dong et al., 2020; Wu et al., 2020c; Zhou et al., 2020). Among other symptoms, patients with COVID-19 present with anosmia, fever, cough, and dyspnea (Parma et al., 2020; Wu et al., 2020b). Unfortunately, specific treatments are still lacking and the scientific community is struggling to develop new therapeutic combinations and discover effective vaccines.

The recently emerged severe acute respiratory syndrome coronavirus 2 (SARS-CoV-2) belongs to the family of betacoronaviruses and contains a single-stranded RNA of approximately $30 \mathrm{~kb}$ in length ( $\mathrm{Wu}$ et al., 2020a). Altogether, the subgroups of coronaviruses were thought to exclusively infect animals ( $\mathrm{Ng}$ and Hiscox, 2020); however, severe acute respiratory syndrome coronavirus (SARS-CoV) and Middle East respiratory syndrome-related coronavirus caused severe respiratory illness in humans in 2002 and 2012, respectively (Peiris et al., 2004; Kupferschmidt, 2014). In particular, SARS-CoV-2 has infected more than 5 million individuals worldwide, causing more than 300,000 deaths at the time of writing. 
The process of SARS-CoV-2 infection begins with the binding of the viral glycoprotein spike $S$ protein to angiotensin-converting enzyme 2 (ACE2), whose receptor is expressed on the human airway epithelium. After fusion occurs, the type II transmembrane serine protease (TMPRSS2) present on the cell surface clears ACE2 and activates the receptor-attached spike-like S protein (Hoffmann et al., 2020), leading to conformational changes necessary for virus entry. After viral RNA release in the cytoplasm, the genomic RNA is translated into the viral replicase polyproteins ppl and 1ab. Then, the polymerase produces a series of subgenomic mRNAs, which are translated into new structural proteins and translocated from the endoplasmic reticulum to the Golgi apparatus, where the new virions are assembled. At this point, there remain significant gaps in our knowledge concerning the host effectors involved in viral replication and infection. Following examination of the SARS-CoV-2 transcriptome (Ferretti et al., 2020; Kim et al., 2020), the use of proteomic strategies has emerged as a valuable tool to cope with this viral sprout. In fact, proteomic methods allowed the characterization and understanding of SARS-CoV during the severe acute respiratory syndrome (SARS) outbreak in 2003, enabling the description of virus protein sequences and antigenic virus proteins and the identification of ACE2 as the human receptor for SARS-CoV (Krokhin et al., 2003; Li et al., 2003; Ying et al., 2004). Consequently, the application of proteomic workflows may be highly beneficial to increase our understanding of the pathogenesis of COVID-19, to identify potential therapeutic targets, and even to develop fast and effective diagnostic tests (Struwe et al., 2020; Whetton et al., 2020). In this mini-review, we focus on different proteomic methodologies currently used to decipher novel SARS-CoV-2 targets and potential COVID-19 biomarkers (Table 1). Given that the nasal cavity is one of the entry sites for SARS-CoV-2, we will also discuss the deployment of olfactory proteomics to unravel cell-signaling networks that are disrupted in olfactory peripheral areas that may potentially explain the early loss of smell observed in patients with COVID-19.

\section{ELUCIDATION OF THE SARS-CoV-2 INTERACTOME}

It is well known that viral proteomes constitute different combinations of protein-protein interactions (PPIs) to accomplish a great variety of vital functions during the viral cycle. However, the study of protein interactions is challenging because of their special physical properties and transitory character. To study protein complexes, a combination of cross-linking and liquid chromatography-tandem mass spectrometry (LC-MS/MS) approaches is commonly used (Haupt et al., 2017), and these techniques have been used recently for the analysis of SARS-CoV-2 PPIs. The $30 \mathrm{~kb}$ SARS-CoV-2 genome encodes for 14 major open reading frames (ORFs) that are further processed into 28 proteins: (i) glycoprotein spike (S), membrane $(\mathrm{M})$, envelope $(\mathrm{E})$, and nucleocapsid $(\mathrm{N})$ structural proteins, (ii) accessory proteins (ORF10, ORF9a, ORF9b, ORF8, ORF7a, ORF7b, ORF6, and ORF3a), and (iii) 16 non-structural proteins (nsp1-nsp16; Wu et al., 2020a). Liang et al. (2020) characterized intraviral PPIs using genome-wide yeast-two hybrid and co-immunoprecipitation, revealing 58 viral PPIs. Interestingly, some viral proteins showed self-association forms, including proteins $\mathrm{M}, \mathrm{N}$, and $\mathrm{E}$, some non-structural proteins (nsp2, nsp5, and nsp8), and some accessory proteins (ORF6, ORF7a, ORF7b, ORF9b, and ORF10), suggesting their important roles in viral replication and immune evasion. In this context, it is important to note that various disadvantages have been addressed in the study of PPIs, such as the lack of essential post-translational modifications for protein interactions that do not occur in yeast or the potential toxicity of some proteins in this system (Van Criekinge and Beyaert, 1999).

Since no specific therapeutics are currently approved for SARS-CoV-2, it is essential to increase knowledge regarding the interaction landscape between viral and human proteins. Taking into account the drawbacks concerning the overexpression of exogenous proteins in cell systems such as protein misfolding, mislocalization, or misregulations (Dunham et al., 2012), the combination of affinity purification and mass spectrometry (AP-LC/MS) represents a feasible approach to obtain high-quality maps of human protein interactomes in a reproducible way (Varjosalo et al., 2013). Gordon et al. (2020) have identified 332 protein interactions between SARSCoV-2 and human proteins. The SARS-CoV-2 human interactome has been shown to impact functions such as DNA replication, epigenetic and gene expression regulation, vesicle trafficking, lipid metabolism, and RNA processing, among others (Gordon et al., 2020). Importantly, SARS-CoV-2 viral proteins target proteins involved in the innate immune system. For instance, Nsp13 interacts with multiple proteins from the transducin-like enhancer of split (TLE) family, which modulates the nuclear factor (NF)- $\mathrm{\kappa B}$ response. Accordingly, interactions between human inflammatory and innate immune key players, such as NF- $\mathrm{kB}$ repressing factor [NKRF; a repressor of interleukin (IL)-8 and IL-6 production], C1QBP (involved in complement $\mathrm{C} 1$ activation), and a great variety of viral non-structural proteins (nsp9, nsp10, etc.), have also been described by Liang et al. (2020) using AP-LC/MS analysis, explaining the high inflammatory response of patients with COVID-19 (Tay et al., 2020). In this case, MS analysis identified 251 human proteins as SARS-CoV-2 interactors. Among others, ribosomal proteins and proteasome-related proteins were identified as viral interactors, indicating alterations in the host gene translation, protein folding, and the degradation pathways. In both studies, the authors overexpressed the SARS-CoV-2 genes with a flag epitope into HEK293 cells. However, the characterized interactome partially differs, probably because of the experimental settings applied (i.e., the timing of cell lysis). Finally, the multilevel proteomic approach described by Stukalov et al. (2020), through the SARS-CoV and SARS-CoV-2 interactome in a lung-derived human cell line (A549), has revealed specific characteristics in the pathogenicity and transmission capabilities of each strain. Moreover, this interactome profiling has allowed the identification of potential targets for SARS-CoV-2 antiviral therapies (Stukalov et al., 2020). 
TABLE 1 | Compilation of recent research using proteomic approaches in a SARS-CoV-2 context.

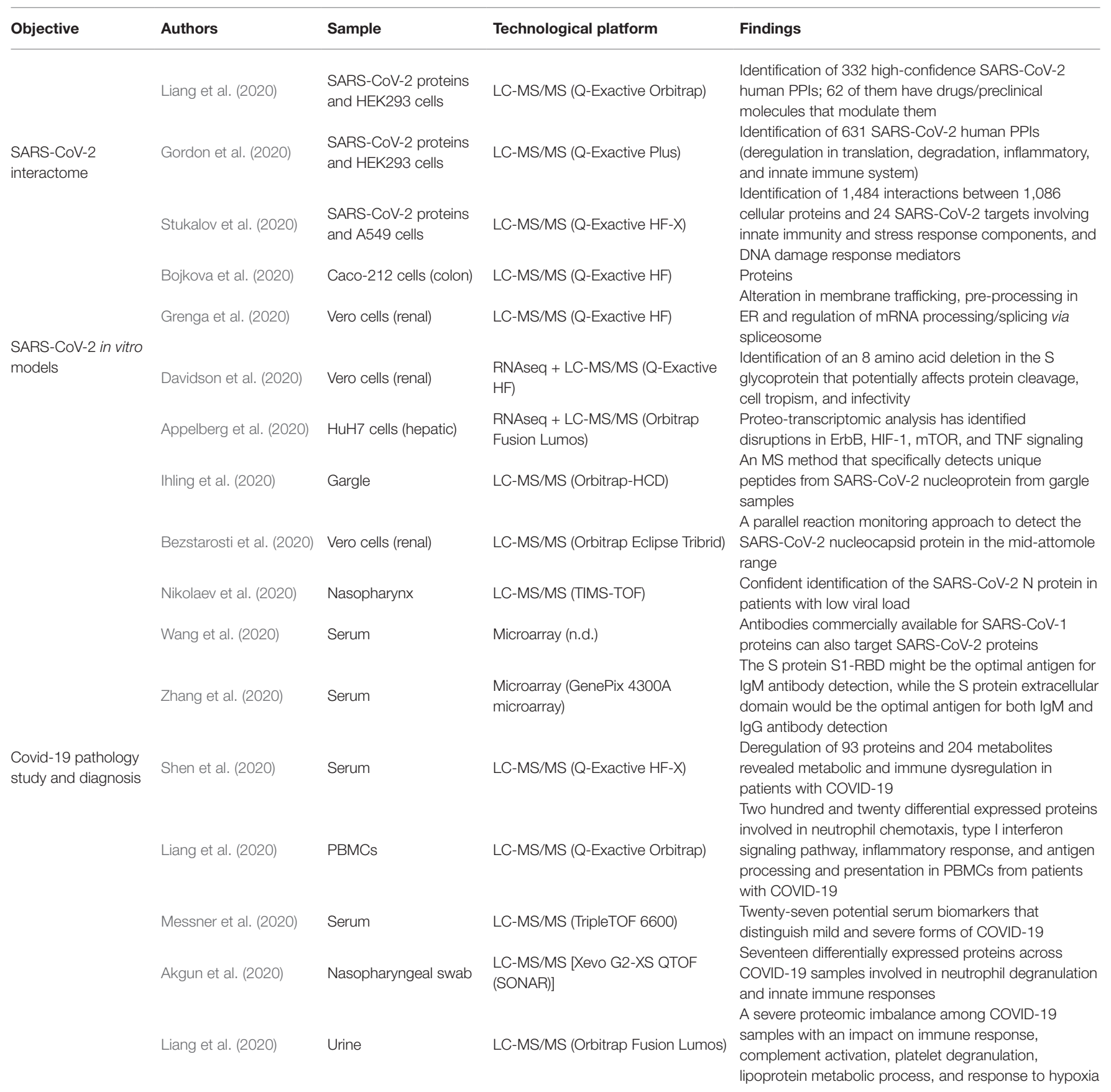

ER, endoplasmic reticulum; ErbB, epidermal growth factor receptor; HIF-1, hypoxia-inducible factor 1; IgG, immunoglobulin G; IgM, immunoglobulin M; LC-MS/MS, liquid chromatography with tandem mass spectrometry; mTOR, mammalian target of rapamycin; n.d., not determined; PBMC, peripheral blood mononuclear cell; PPI, protein-protein interaction; S1-RBD, subunit 1 receptor binding domain; TIMS-TOF, trapped ion mobility spectrometry-time of flight; TNF, tumor necrosis factor.

\section{PROTEOMIC PROFILING OF SARS-CoV-2-INFECTED HOST CELLS}

High-throughput techniques have also been applied to increase biological knowledge of the molecular imbalance triggered by SARS-CoV-2 infection using in vitro models. Analyzing the quantitative translatome and proteome profiles of the Caco-2 cellular response on infection with SARS-CoV-2
(Bojkova et al., 2020), only minor changes were observed at early infection time points. However, alterations in spliceosome components and carbon metabolism were observed at $24 \mathrm{~h}$ post infection (h.p.i.). In this context, the authors proposed the use of specific inhibitors of both pathways as potential therapeutic agents for SARS-CoV-2 infection. Consistent with these data, alterations in the same biological routes together with deregulation in protein pre-processing, vacuole 
formation, and viral budding routes have been detected in SARS-CoV-2-infected Vero cells (Grenga et al., 2020). Moreover, multi-omic characterization at the level of the complete genome, proteome, and phosphoproteome was performed using the same cell line, revealing new and critical aspects of SARSCoV-2 glycoprotein S (Davidson et al., 2020). Finally, Appelberg et al. (2020) combined two high-throughput techniques analyzing the transcriptome and proteome of Huh7 cells upon SARS-CoV-2 infection at 24, 48, and 72 h.p.i. Together with alterations in the thrombotic and metabolic pathways (complement cascades and glycolysis), the mammalian target of rapamycin (mTOR)/hypoxia-inducible factor 1 (HIF-1) signaling route was proposed as a potential therapeutic target against SARS-CoV-2 infection (Appelberg et al., 2020). All differential molecules characterized in SARS-CoV-2-infected cells have extended our knowledge about the metabolic imbalance induced during the viral infection. However, the molecular repertoires differ significantly across studies. This variability is likely associated with the experimental designs applied (different origin of the cell lines, multiplicity of infection, and time course used) and the heterogeneity of the analytical workflows used (protein precipitation, digestion and fractionation protocols, and MS workflows; Table 1). Although differential proteotyping has contributed to a better understanding about SARS-CoV-2 infection, more efforts are needed to decipher whether the differentially expressed proteins may be considered SARS-CoV-2 targets involved in the modulation of the host metabolism or are part of the molecular events triggered by the cell to interfere with viral propagation.

\section{CLINICAL PROTEOMICS IN COVID-19}

Although the genome/proteome characterization of SARS-CoV-2 provides valuable information regarding the virus structure and protein interactions, understanding how the infection alters the metabolic homeostasis in patients with COVID-19 may provide resources for novel diagnostic and therapeutic interventions. The diagnostic potential of proteomics has already been demonstrated by a shotgun proteomic workflow, in which SARSCoV-2 unique peptides were identified in gargle samples from patients with COVID-19 (Ihling et al., 2020) and SARS-CoV-2 peptides have been identified in the mid-attomole range by parallel reaction monitoring (Bezstarosti et al., 2020). Moreover, a rapid MS protocol has been recently developed to detect peptides corresponding to the viral nucleocapsid $\mathrm{N}$ protein from nasopharyngeal epithelial swab samples in less than $1 \mathrm{~h}$ (Nikolaev et al., 2020). In order to improve serology tests for rapid COVID-19 screening, antibody microarrays detecting SARSCoV-2 antigens have also been developed, mapping the humoral response of patients with COVID-19 (Wang et al., 2020; Zhang et al., 2020). Also, Shen et al. (2020) have combined isobaric proteomics and untargeted metabolomics with the aim of analyzing molecular perturbances occurring in the sera of patients with SARS-CoV-2. This study has shown a dysregulation in lipid metabolism due to changes in multiple apolipoproteins and steroid hormones, and activation of acute-phase proteins and the complement system. On the other hand, Liang et al. (2020) performed a labeling quantitative LC-MS/MS analysis in peripheral blood mononuclear cells from patients with COVID-19, revealing an imbalance in neutrophil activation, type I interferon signaling, inflammation, and antigen processing (Liang et al., 2020). Accordingly, recent proteomic studies have also demonstrated alterations in neutrophil and platelet degranulation and the immune system response within nasopharyngeal and urine samples derived from patients with COVID-19 (Akgun et al., 2020; Li et al., 2020). These results confirm the clinical data evidencing an innate immune response and subsequent cytokine storm during SARS-CoV-2 infection (Tay et al., 2020). Moreover, Messner et al. (2020) have demonstrated the utility of MS-based methods in clinical practice, reporting 27 potential serum biomarkers for COVID-19. Interestingly, these authors have applied short gradients combined with data-independent acquisition (SWATH-MS), achieving precise, reproducible, robust, and low-cost results, allowing 180 samples per day on a single mass spectrometer. Based on these studies, it is evident that the sensitivity and high throughput of specific MS approaches may complement the current clinical genetic studies based on the polymerase chain reaction, being a technological pillar to be considered for future assistance in tackling the worldwide spread of the novel coronavirus causing COVID-19.

\section{EFFECTS OF SARS-CoV-2 IN CHEMOSENSORY PROCESSING: WHAT ROLE CAN PROTEOMICS PLAY?}

Emerging evidence shows that SARS-CoV-2 infection causes pleiotropic effects. Apart from the respiratory system, other tissues such as the small intestine, renal tubules, and arterial smooth muscle cells are vulnerable to SARS-CoV-2 infection because of their high expression of ACE2 (Zou et al., 2020). Among other symptoms, olfactory loss is the early predominant neurological symptom (Baig et al., 2020; Lechien et al., 2020; Moein et al., 2020; Parma et al., 2020). The nasal cavity is one of the routes of SARS-CoV-2 entry, where the presence of ACE2 and TMPRSS2 in stem cells and olfactory epithelial support cells is higher than that in olfactory sensory neurons (Brann et al., 2020; Ueha et al., 2020), suggesting that the first group may be partially responsible for the olfactory dysfunction observed in patients with COVID-19. Both ACE2 and TMPRSS2 genes are co-expressed with genes involved in innate immunity in nasal structures, indicating a potential role in battling the infection in the initial phases of the disease (Sungnak et al., 2020). Nevertheless, despite all this evidence, the molecular consequences of the entrance of the virus in the olfactory system remain unknown. Owing to the relevance of olfactory loss as an early predictor of COVID-19, the application of olfactory proteomics (Lachén-Montes et al., 2016) would shed new light on the potential mechanisms disrupted by SARS-CoV-2 at the level of: (i) the olfactory neuroepithelium (involved in odor perception), (ii) the olfactory bulbs and tracts (involved in the transmission of olfactory information), and (iii) the olfactory cortex (involved in odor recognition/ 


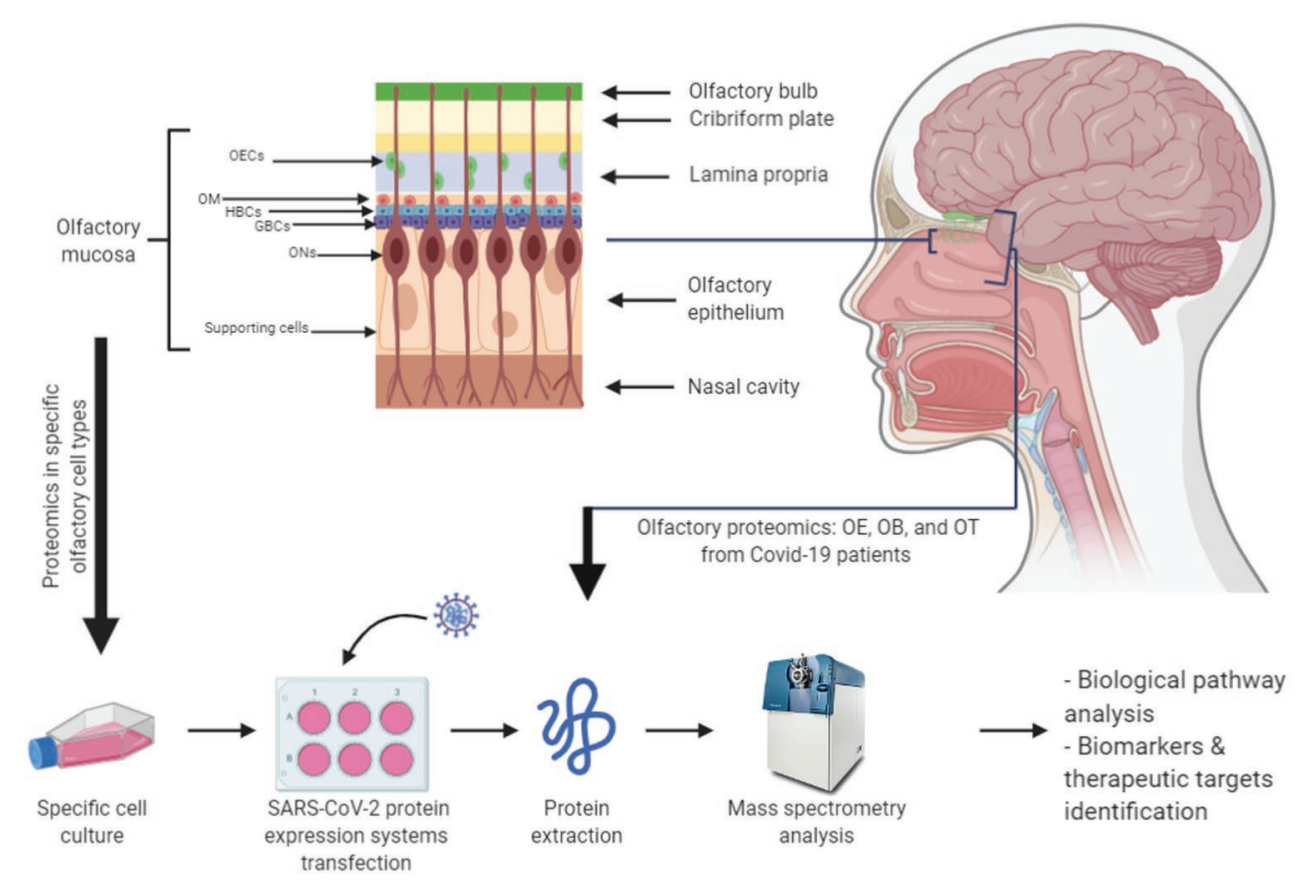

FIGURE 1 | Potential olfactory proteomics workflows to characterize molecular disturbances caused by severe acute respiratory syndrome coronavirus 2 (SARSCoV-2). The combination of commercial or primary cell lines from the olfactory mucosa with the transfection of expression systems for SARS-CoV-2 proteins (or infection with viral particles) represents a valuable approach to study the impact of SARS-CoV-2 infection in this region. In addition, the proteomic analysis of the OE-OB-OT axis from patients with COVID-19 will provide novel insights into the olfactory dysfunction triggered by SARS-CoV-2. GBC, globular basal stem cell; HBC, horizontal basal stem cell; OB, olfactory bulb; OE, olfactory epithelium; OEC, olfactory ensheathing cell; OM, olfactory mucosa mesenchymal stem cell; ON, olfactory neuron; OT, olfactory tract.

discrimination and memorization). To do this, the scientific community requires autopsies of the brains of patients with COVID-19, detailed neuropathological examination, and initiatives aimed at biobanking of the olfactory epithelium and olfactory structures. However, for biosecurity reasons, the availability of this biological material is not well established. To partially solve this problem, the deployment of highly controlled in vitro systems that model different stations of the olfactory pathway in combination with large-scale MS-based quantitative proteomics will allow the molecular imbalance induced by SARS-CoV-2 in cellular and neuronal circuits in the central olfactory areas to be monitored (Figure 1).

\section{FUTURE DIRECTIONS}

The urgent need for the scientific community to face the current SARS-CoV-2 world pandemic has evidenced the great efforts of proteomic scientists to decipher molecular knowledge about SARSCoV-2 and COVID-19. In this mini-review, we have proposed analysis of the intranasal route of SARS-CoV-2 between the nose and the brain to improve our understanding of the early olfactory loss observed in patients with COVID-19. The application of single-cell proteomics or laser capture microdissection coupled with LC-MS/MS analysis will shed new light on the vulnerability of specific olfactory cell types through the specific characterization of deregulated proteomic maps triggered by SARS-CoV-2 infection. Alternative approaches simulating SARS-CoV-2 infection in olfactory cell lines could also be useful to study the progression of the viral infection using a wide range of proteomic strategies, identifying new viral-host protein interactions and potential therapeutic targets for non-invasive intranasal treatments. However, urgent and conjoined efforts from researchers, clinicians, and biosafety and ethics authorities are needed to facilitate the provision of clinical and autopsy biospecimens in order to clarify and understand the potential alterations that occur in the olfactory axis during COVID-19 progression.

\section{AUTHOR CONTRIBUTIONS}

ES: conceptualization. ML-M, FC, JF-I, and ES: formal analysis. FC, JF-I, and ES: funding acquisition. ES: supervision. ML-M and ES: writing - original draft. All authors contributed to the article and approved the submitted version.

\section{FUNDING}

This work was funded by a grant from the Spanish National Research Council (CSIC) with reference PIE-CSIC-202020E108. The Navarrabiomed and Centro Nacional de Biotecnología 
Proteomics Platforms are members of Proteored [PRB3-(Instituto de Salud Carlos III)] and are supported by grant no. PT17/0019/009 to FC and JF-I, of the PE I+D+I 2013-2016 funded by ISCIII and FEDER. The Clinical Neuroproteomics Unit is a member

\section{REFERENCES}

Akgun, E., Tuzuner, M. B., Sahin, B., Kilercik, M., Kulah, C., Cakiroglu, H. N., et al. (2020). Altered molecular pathways observed in naso-oropharyngeal samples of SARS-CoV-2 patients. medRxiv [Preprint]. doi: 10.1101/2020.05.14.20102558

Appelberg, S., Gupta, S., Ambikan, A. T., Mikaeloff, F., Végvári, Á., Akusjärvi, S. S., et al. (2020). Dysregulation in mTOR/HIF-1 signaling identified by proteotranscriptomics of SARS-CoV-2 infected cells. bioRxiv [Preprint]. doi: 10.1101/2020.04.30.070383

Baig, A. M., Khaleeq, A., Ali, U., and Syeda, H. (2020). Evidence of the COVID-19 virus targeting the CNS: tissue distribution, host-virus interaction, and proposed neurotropic mechanisms. ACS Chem. Neurosci. 11, 995-998. doi: 10.1021/acschemneuro.0c00122

Bezstarosti, K., Lamers, M. M., Haagmans, B. L., and Demmers, J. A. (2020). Targeted proteomics for the detection of SARS-CoV-2 proteins. bioRxiv [Preprint]. doi: 10.1101/2020.04.23.057810

Bojkova, D., Klann, K., Koch, B., Widera, M., Krause, D., Ciesek, S., et al. (2020). SARS-CoV-2 infected host cell proteomics reveal potential therapy targets. [Preprint]. doi: 10.21203/rs.3.rs-17218/v1

Brann, D. H., Tsukahara, T., Weinreb, C., Logan, D. W., and Datta, S. R. (2020). Non-neural expression of SARS-CoV-2 entry genes in the olfactory epithelium suggests mechanisms underlying anosmia in COVID-19 patients. bioRxiv [Preprint]. doi: 10.1101/2020.03.25.009084

Davidson, A. D., Williamson, M. K., Lewis, S., Shoemark, D., Carroll, M. W., Heesom, K., et al. (2020). Characterisation of the transcriptome and proteome of SARS-CoV-2 using direct RNA sequencing and tandem mass spectrometry reveals evidence for a cell passage induced in-frame deletion in the spike glycoprotein that removes the furin-like cleavage site. bioRxiv [Preprint]. doi: 10.1101/2020.03.22.002204

Dong, E., Du, H., and Gardner, L. (2020). An interactive web-based dashboard to track COVID-19 in real time. Lancet Infect. Dis. 20, 533-534. doi: 10.1016/ S1473-3099(20)30120-1

Dunham, W. H., Mullin, M., and Gingras, A. C. (2012). Affinity-purification coupled to mass spectrometry: basic principles and strategies. Proteomics 12, 1576-1590. doi: 10.1002/pmic.201100523

Ferretti, L., Wymant, C., Kendall, M., Zhao, L., Nurtay, A., Abeler-Dörner, L., et al. (2020). Quantifying SARS-CoV-2 transmission suggests epidemic control with digital contact tracing. Science 368:eabb6936. doi: 10.1126/science.abb6936

Gordon, D. E., Jang, G. M., Bouhaddou, M., Xu, J., Obernier, K., O’Meara, M. J., et al. (2020). A SARS-CoV-2-human protein-protein interaction map reveals drug targets and potential drug-repurposing. bioRxiv [Preprint]. doi: 10.1101/2020.03.22.002386

Grenga, L., Gallais, F., Pible, O., Gaillard, J. -C., Gouveia, D., Batina, H., et al. (2020). Shotgun proteomics of SARS-CoV-2 infected cells and its application to the optimisation of whole viral particle antigen production for vaccines. bioRxiv [Preprint]. doi: 10.1101/2020.04.17.046193

Haupt, C., Hofmann, T., Wittig, S., Kostmann, S., Politis, A., and Schmidt, C. (2017). Combining chemical cross-linking and mass spectrometry of intact protein complexes to study the architecture of multi-subunit protein assemblies. J. Vis. Exp. 56747. doi: 10.3791/56747

Hoffmann, M., Kleine-Weber, H., Schroeder, S., Krüger, N., Herrler, T., Erichsen, S., et al. (2020). SARS-CoV-2 cell entry depends on ACE2 and TMPRSS2 and is blocked by a clinically proven protease inhibitor. Cell 181, 271.e8-280.e8. doi: $10.1016 /$ j.cell.2020.02.052

Ihling, C., Tänzler, D., Hagemann, S., Kehlen, A., Hüttelmaier, S., and Sinz, A. (2020). Mass spectrometric identification of SARS-CoV-2 proteins from gargle solution samples of COVID-19 patients. bioRxiv [Preprint]. doi: 10.1101/2020.04.18.047878

Kim, D., Lee, J. Y., Yang, J. S., Kim, J. W., Kim, V. N., and Chang, H. (2020). The architecture of SARS-CoV-2 transcriptome. Cell 181, 914.e10-921.e10. doi: $10.1016 /$ j.cell.2020.04.011 of the Global Consortium for Chemosensory Research (GCCR) and the Spanish Olfactory Network (ROE; supported by grant no. RED2018-102662-T, funded by Spanish Ministry of Science and Innovation).

Krokhin, O., Li, Y., Andonov, A., Feldmann, H., Flick, R., Jones, S., et al. (2003). Mass spectrometric characterization of proteins from the SARS virus: a preliminary report. Mol. Cell. Proteomics 2, 346-356. doi: 10.1074/mcp. M300048-MCP200

Kupferschmidt, K. (2014). Emerging diseases: soaring MERS cases in Saudi Arabia raise alarms. Science 344, 457-458. doi: 10.1126/science.344.6183.457

Lachén-Montes, M., Fernández-Irigoyen, J., and Santamaría, E. (2016). Deconstructing the molecular architecture of olfactory areas using proteomics. Proteomics Clin. Appl. 10, 1178-1190. doi: 10.1002/prca.201500147

Lechien, J. R., Chiesa-Estomba, C. M., De Siati, D. R., Horoi, M., Le Bon, S. D., Rodriguez, A., et al. (2020). Olfactory and gustatory dysfunctions as a clinical presentation of mild-to-moderate forms of the coronavirus disease (COVID-19): a multicenter European study. Eur. Arch. Otorhinolaryngol. 277, 2251-2261. doi: 10.1007/s00405-020-05965-1

Li, W., Moore, M. J., Vasllieva, N., Sui, J., Wong, S. K., Berne, M. A., et al. (2003). Angiotensin-converting enzyme 2 is a functional receptor for the SARS coronavirus. Nature 426, 450-454. doi: 10.1038/nature02145

Li, Y., Wang, Y., Liu, H., Sun, W., Ding, B., Zhao, Y., et al. (2020). Urine proteome of COVID-19 patients. medRxiv [Preprint]. doi: 10.1101/ 2020.05.02.20088666

Liang, Q., Li, J., Guo, M., Tian, X., Liu, C., Wang, X., et al. (2020). Virus-host interactome and proteomic survey of PMBCs from COVID-19 patients reveal potential virulence factors influencing SARS-CoV-2 pathogenesis. bioRxiv [Preprint]. doi: 10.1101/2020.03.31.019216

Messner, C. B., Demichev, V., Wendisch, D., Michalick, L., White, M., Freiwald, A., et al. (2020). Clinical classifiers of COVID-19 infection from novel ultra-high-throughput proteomics. medRxiv [Preprint]. doi: 10.1101/ 2020.04.27.20081810

Moein, S. T., Hashemian, S. M. R., Mansourafshar, B., Khorram-Tousi, A., Tabarsi, P., and Doty, R. L. (2020). Smell dysfunction: a biomarker for COVID-19. Int. Forum Allergy Rhinol. 10, 944-950. doi: 10.1002/alr.22587

Ng, L. F. P., and Hiscox, J. A. (2020). Coronaviruses in animals and humans. BMJ 368:m634. doi: 10.1136/bmj.m634

Nikolaev, E. N., Indeykina, M. I., Brzhozovskiy, A. G., Bugrova, A. E., Kononikhin, A. S., Starodubtseva, N. L., et al. (2020). Mass spectrometric detection of SARS-CoV-2 virus in scrapings of the epithelium of the nasopharynx of infected patients via nucleocapsid $\mathrm{N}$ protein. bioRxiv [Preprint]. doi: 10.1101/2020.05.24.113043

Parma, V., Ohla, K., Veldhuizen, M. G., Niv, M. Y., Kelly, C. E., Bakke, A. J., et al. (2020). More than just smell. COVID-19 is associated with severe impairment of smell, taste, and chemesthesis. medRxiv [Preprint]. doi: 10.1101/2020.05.04.20090902

Peiris, J. S. M., Guan, Y., and Yuen, K. Y. (2004). Severe acute respiratory syndrome. Nat. Med. 10, S88-S97. doi: 10.1038/nm1143

Shen, B., Yi, X., Sun, Y., Bi, X., Du, J., Zhang, C., et al. (2020). Proteomic and metabolomic characterization of COVID-19 patient sera. SSRN Electron. J. [Preprint]. doi: 10.2139/ssrn.3570565

Struwe, W., Emmott, E., Bailey, M., Sharon, M., Sinz, A., Corrales, F. J., et al. (2020). The COVID-19 MS coalition-accelerating diagnostics, prognostics, and treatment. Lancet 395, 1761-1762. doi: 10.1016/S0140-6736(20)31211-3

Stukalov, A., Girault, V., Grass, V., Bergant, V., Karayel, O., Urban, C., et al. (2020). Multi-level proteomics reveals host-perturbation strategies of SARSCoV-2 and SARS-CoV. bioRxiv [Preprint]. doi: 10.1101/2020.06.17.156455

Sungnak, W., Huang, N., Bécavin, C., Berg, M., Queen, R., Litvinukova, M., et al. (2020). SARS-CoV-2 entry factors are highly expressed in nasal epithelial cells together with innate immune genes. Nat. Med. 26, 681-687. doi: 10.1038/ s41591-020-0868-6

Tay, M. Z., Poh, C. M., Rénia, L., MacAry, P. A., and Ng, L. F. P. (2020). The trinity of COVID-19: immunity, inflammation and intervention. Nat. Rev. Immunol. 20, 363-374. doi: 10.1038/s41577-020-0311-8

Ueha, R., Kondo, K., Kagoya, R., Shichino, S., Ueha, S., and Yamasoba, T. (2020). Background mechanisms of olfactory dysfunction in COVID-19: 
expression of ACE2, TMPRSS2, and Furin in the nose and olfactory bulb in human and mice. bioRxiv [Preprint]. doi: 10.1101/2020.05.15.097352

Van Criekinge, W., and Beyaert, R. (1999). Yeast two-hybrid: state of the art. Biol. Proced. Online 2, 1-38. doi: 10.1251/bpo16

Varjosalo, M., Sacco, R., Stukalov, A., Van Drogen, A., Planyavsky, M., Hauri, S., et al. (2013). Interlaboratory reproducibility of large-scale human proteincomplex analysis by standardized AP-MS. Nat. Methods 10, 307-314. doi: $10.1038 /$ nmeth. 2400

Wang, H., Hou, X., Wu, X., Liang, T., Zhang, X., Wang, D., et al. (2020). SARS-CoV-2 proteome microarray for mapping COVID-19 antibody interactions at amino acid resolution. bioRxiv [Preprint]. doi: 10.1101/ 2020.03.26.994756

Whetton, A. D., Preston, G. W., Abubeker, S., and Geifman, N. (2020). Proteomics and informatics for understanding phases and identifying biomarkers in COVID-19 disease. J. Proteome Res. doi: 10.1021/acs.jproteome.0c00326 [Epub ahead of print]

Wu, A., Peng, Y., Huang, B., Ding, X., Wang, X., Niu, P., et al. (2020a). Genome composition and divergence of the novel coronavirus (2019-nCoV) originating in China. Cell Host Microbe. 27, 325-328. doi: 10.1016/j. chom.2020.02.001

Wu, D., Wu, T., Liu, Q., and Yang, Z. (2020b). The SARS-CoV-2 outbreak: what we know. Int. J. Infect. Dis. 94, 44-48. doi: 10.1016/j.ijid.2020.03.004

Wu, F., Zhao, S., Yu, B., Chen, Y. M., Wang, W., Song, Z. G., et al. (2020c). A new coronavirus associated with human respiratory disease in China. Nature 579, 265-269. doi: 10.1038/s41586-020-2008-3
Ying, W., Hao, Y., Zhang, Y., Peng, W., Qin, E., Cai, Y., et al. (2004). Proteomic analysis on structural proteins of Severe Acute Respiratory Syndrome coronavirus. Proteomics 4, 492-504. doi: 10.1002/pmic.200300676

Zhang, X., Wu, X., Wang, D., Lu, M., Hou, X., Wang, H., et al. (2020). Proteomewide analysis of differentially-expressed SARS-CoV-2 antibodies in early COVID-19 infection. medRxiv [Preprint]. doi: 10.1101/2020.04.14.20064535

Zhou, P., Yang, X. L., Wang, X. G., Hu, B., Zhang, L., Zhang, W., et al. (2020). A pneumonia outbreak associated with a new coronavirus of probable bat origin. Nature 579, 270-273. doi: 10.1038/s41586-020-2012-7

Zou, X., Chen, K., Zou, J., Han, P., Hao, J., and Han, Z. (2020). Single-cell RNA-seq data analysis on the receptor ACE2 expression reveals the potential risk of different human organs vulnerable to 2019-nCoV infection. Front. Med. 14, 185-192. doi: 10.1007/s11684-020-0754-0

Conflict of Interest: The authors declare that the research was conducted in the absence of any commercial or financial relationships that could be construed as a potential conflict of interest.

Copyright (c) 2020 Lachén-Montes, Corrales, Fernández-Irigoyen and Santamaría. This is an open-access article distributed under the terms of the Creative Commons Attribution License (CC BY). The use, distribution or reproduction in other forums is permitted, provided the original author(s) and the copyright owner(s) are credited and that the original publication in this journal is cited, in accordance with accepted academic practice. No use, distribution or reproduction is permitted which does not comply with these terms. 